\title{
Prevalence of Congenitally Missing Permanent Teeth in a Group of Yemeni Population: A Radiographic Study
}

\author{
Ziad A. N. Musaed ${ }^{1 *}$, Ghamdan Al-Harazi ${ }^{2}$, Hussein Shuga Al-Deen
}

${ }^{1}$ Orthodontics MSc Candidate, Department of orthodontics, Pedodontics and Preventive Dentistry, Faculty of Dentistry, Sana'a University, Yemen

${ }^{2}$ Professor of orthodontics, Department of orthodontic, Pedodontic, and preventive dentistry, Faculty of Dentistry, Sana'a University, Yemen

${ }^{3}$ Assistant Professor of Pedodontics, Department of orthodontics, Pedodontics and Preventive Dentistry, Faculty of Dentistry, Sana'a University, Yemen

DOI: $10.36348 /$ SJODR.2019.v04i10.004

| Received: 08.10.2019| Accepted: 14.10.2019| Published: 22.10.2019

*Corresponding author: Ziad A. N. Musaed

\section{Abstract}

Objective: This study aims to estimate the prevalence of congenitally missing permanent teeth in a group of 5100 dental patients in Yemen. Methods: A total of 5100 digital panoramic radiographs were retrospectively examined for the presence of congenitally missing permanent teeth. The radiographs were obtained from the archival records of patients attending a local dental centers, and colleges of dentistry at the provinces of Ibb, Thamar, and Sana'a in Yemen. Results: A total of 293 congenitally missing teeth were observed in 165patients (136 females and 29males); the overall prevalence of congenitally missing teeth in permanent dentition was $3.23 \%$ (2.23\% in males, $3.58 \%$ in females). Congenitally missing teeth was more prevalent in the maxilla (55\%) than in the mandible (45\%) and in the right side of the jaws $(52.6 \%)$ than in the left side $(47.4 \%)$. The most common congenitally missing permanent tooth was the maxillary lateral incisor $(44.48 \%)$, followed by the mandibular 2nd premolar $(34.48 \%)$, the maxillary 2nd premolar (15.51\%), the mandibular lateral incisor $(5.17 \%)$, and the maxillary canine $(0.7 \%)$. Certain anomalies were co-existed along with tooth agenesis in $66(40 \%)$ patients. Canine impaction was the most common among 29 (17.58\%) patients followed by microdontia among $27(16.37 \%)$ patients. Other anomalies present were transposed teeth among $4(2.43 \%)$ patients, ectopic eruption among 42.43$) \%$ ) patients and supernumerary among $2(1.21 \%)$ patients. Canine impaction was commonly seen in relation to the lateral incisor agenesis followed by microdontia. The present study results give a clue of the magnitude of the problem, but further studies are required to identify the etiology of dental agenesis in Yemen.

Keywords: Agenesis, Congenitally missing teeth, Hypodontia, Tooth absence.

Copyright @ 2019: This is an open-access article distributed under the terms of the Creative Commons Attribution license which permits unrestricted use, distribution, and reproduction in any medium for non-commercial use (NonCommercial, or CC-BY-NC) provided the original author and source are credited.

\section{INTRODUCTION}

Dental treatments are rather expensive health services and the combination of different modalities such as orthodontic, prosthodontics, and surgical treatments can put a heavy burden on the average family's health budget. Some frequent dental anomalies need quite expensive treatments. One of them is congenitally missing teeth (CMT), congenital absence of teeth, congenital dental aplasia, or dental agenesis [1].

Congenitally missing teeth is the most common developmental dental anomalies in humans [2]. Tooth agenesis refers to the failure of a tooth formation and could be classified into anodontia or partial anodontia [3]. Anodontia is the total absence of tooth development in primary, permanent or both dentitions. Partial anodontia is the lack of development and absence of one or more teeth. Partial hypodontia may be further subdivided into oligodontia which is the congenital absence of more than six teeth (third molars are not included) and hypodontia which is the congenital absence of one to six teeth (third molars are not included) [2].

Developmental dental anomalies can be diagnosed clinically and treatment planning is important for such reasons: anomalies are often associated with some problems such as caries, poor oral hygiene, orthodontic and aesthetic concerns. In addition, when these anomalies are observed, especially if they are multiple, some syndromes may be considered. For such reasons the prevalance and distribution of dental anomalies become important. Also the prevalence of dental anomalies can provide important informaton for both the anthropological and clinical management of patients, and are essential to understanding the diferences within and between populations [4]. Many studies have been carried out on the frequency of dental agenesis in different populations and the data provided 
so far for tooth agenesis in either genders varies between 0.3 per cent and 11.3 per cent, excluding the third molars [5].

A meta-analysis made by Polder [6] in 2004, showed that the dental agenesis is usually 1.37 times more frequent in females than in males. The missing teeth were more often absent on the maxillary arch than on the mandibular arch [7] and on the right side than on the left side within the dental arch [5]. Although any tooth can be susceptible to agenesis, lateral incisors and second premolars show a great probability of agenesis [6]. The most common teeth reported missing varies among different ethnicities. The maxillary lateral incisors were the most common in the Malaysian [5], Turkish [9], Romanian [10], Spanish [11], and American [12] populations, while the mandibular second premolars were the most common in the the Jordanian [13], Iraqi [14], Indian [15] and European [6] populations. However, a higher incidence of missing mandibular incisors is observed in Chinese [16], Korean [17], and Japanese [18] populations than in Caucasian populations.

Teeth develop from the dental lamina, and then commence interactions with the epithelia and underlying mesenchyme. As tooth development advances, enamel knots mediate crown size and cusp formation [19]. Dental anomalies can occur due to disturbance of these processes by genetic factors, environmental (local or systemic) factors, or both [20]. It is reported that local factors may lead to congenitally missing teeth depending on the reasons such as squeezing in germ formation in the relevant region, ruptures occurring in dental lamina, the lack of space in the region, functional anomalies in dental epithelium, problems occurring in the formation of mesenchymal tissue or the ruptures occurring during embryonic fusion of the upper jaw and the medial nasal process [21]. With respect to environmental factors, it is reported that trauma, chemotherapy, radiotherapy, thalidomide derivative drugs by mother during her pregnancy and insufficient nourishment of the mother, trauma, infections (rubella, syphilis), osteomyelitis, radiation and hormonal changes may cause congenitally missing teeth [22]. Dental agenesis can also occur as a symptom of more generalized systemic conditions such as ectodermal dysplasia, cleft lip and palate, Down syndrome, Oto-palato-digital syndrome, Ocluo-Facial-Cardio Syndrome, and Reigre syndrome $[23,22]$. Although tooth agenesis is caused by environmental factors in the majority of cases, it has a genetic basis. A familial hypodontia is an autosomal dominant inheritance with incomplete penetrance and variable expressivity. An autosomal recessive mode of inheritance is also possible [24, 25].

Tooth agenesis is often accompanied by other tooth anomalies such as microdontia, ectopic eruption, and impaction [26]. According to Ben-Bassat and Brin
[27], it has been suggested that patients with congenitally missing teeth have specific characteristics of craniofacial morphology and growth patterns. Also, they have found that patients with congenitally missing teeth had a shorter maxilla, a more prognathic mandible, a smaller mandibular plane angle, and greater retroclination of the maxillary and mandibular incisors, and these characteristics were reported to be affected by the severity of congenitally missing teeth, and are probably caused by underdevelopment of the apical base due to the absence of tooth buds. Also, it was reported that this dental agenesis may cause disorders in speech, aesthetic and muscle functions in people [28].

Recent studies realized that hypodontia may be an indicator of susceptibility to developing cancer [9]. Hypodontia was reported more common [8:1] in women with epithelial ovarian cancer than women without epithelial ovarian cancer [29].

In orthodontics, hypodontia due to agenesis of certain teeth greatly affects a patient's function and aesthetic, especially the upper lateral incisors [30]. The incidence of hypodontia in the anterior segments requires great need for orthodontic and prosthodontics. Orthodontic treatment should be in harmony with modern medical thought: "It is better to prevent than to cure". We should not treat the symptom; we should treat the cause. Therefore, early recognition of a tooth agenesis is helpful in order to provide good treatment and prevent a developing malocclusion, also with early detection of hypodontia, alternative treatment modalities can be planned and performed with multidisciplinary team approach restoring the esthetic and function [31].

The treatment options available for cases with congenitally missing teeth are the maintenance of the primary teeth, orthodontic space closure, and space maintenance, restoration with adhesive or fixed denture, tooth transplantation, dental implant or orthodontics space redistribution to facilitate the prosthetic treatment [32].

People with multiple missing teeth deserve the same opportunities for getting a good oral health and appearance as the other human beings [33], therefore, to improve oral health in a population we should begin with collection of data which will help us to understand the needs of the community, to identify high risk groups, and to plan treatment and prevention strategies and monitor the development of the situation over a period of several years [34].

With regard to Yemeni population, the result of a PubMed search was conducted in January 2018 about the prevalence of missing permanent teeth revealed no published data for them. Also, with the recent fast-growing community demand for orthodontic treatment and the paucity of pertinent orthodontic data, 
such information related to clinical orthodontic practice in Yemen is of vital importance and needs to be addressed. Therefore, the current study will be designed to create baseline information on the prevalence and distribution of congenitally missing permanent teeth among a group of Yemeni population (in relation to the site, jaw, tooth type and gender.), to record the associated dental anomalies, and to draw attention to pediatric dentist-pediatrician cooperation in the early diagnosis of congenitally missing teeth

\section{MATERIALS AND METHODS \\ Study sample}

A total number of 5100 panoramic radiographs for Yemeni dental patients ( 3800 females (74.5\%) and $1300(25.5 \%)$ males) aged between 9 and 25 yearsthe ) (mean age was 15 yearswere collected from the archives of the Faculties of dentistry, and private dental clinics in Sana'a, Ibb, and Thamar provinces, Yemen between May 2011 and October 2018.

Table-1: Gender distribution of sample population

\begin{tabular}{|l|l|l|}
\hline Gender & No. & \% \\
\hline Male & 1300 & 25.5 \\
\hline Female & 3800 & 75.5 \\
\hline Total & 5100 & 100 \\
\hline
\end{tabular}

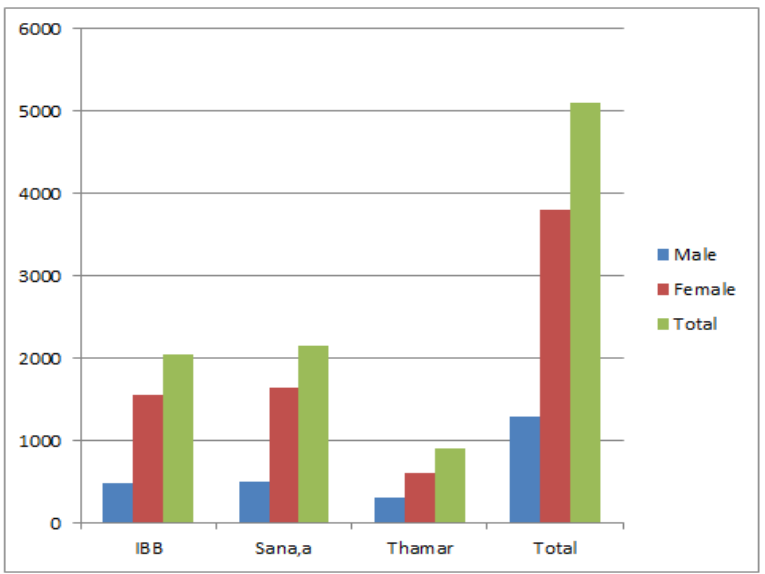

Fig-1: Number and distribution of sample population

\section{type Study}

The present study was a cross sectional retrospective descriptive study conducted based on the clinical records and panoramic radiographs of the patients who attended to the dental clinics for evaluation of their dental problems.

\section{Inclusion and exclusion criteria}

The inclusion criteria for this study were patients of Yemeni origin, patients with no history of medical problems, patients with no history of any syndrome, presence of high quality panoramic radiograph (with proper record of name, date of birth and sex), and patients between 9 and 25 years of age. The exclusion criteria were patients with missing teeth for decay processes, avulsions or extracted for orthodontics or other reasons, panoramic radiography of Non-Yemeni patients, patients with facial clefts and craniofacial syndromes, and poor image quality of panoramic radiographs. Study protocol and ethical approval.

The operator analyzed the available records, the medical history, and the panoramic images of the patients, considering the exclusion and inclusion criteria, to identify the presence of dental agenesis (excluding third molar). The panoramic images were examined by two experienced examiners in a standardized manner under good lighting conditions. The absence of a tooth was considered congenital, if it did not show up on the radiograph (the mineralization of its crown could not be identified on the radiograph), and anamnestic data confirmed that the tooth was not extracted or lost by trauma. Ethical approval for the study would be approved by the Research Committee of the faculties of dentistry Sanaa, Ibb, and Thamar universities.

\section{STATISTICAL ANALYSIS}

Statically analysis was performed using statistical package software system, version 13 (SPSS 13.0®), and descriptive statistical analysis (mean, standard deviation) will be calculated. Pearsons chisquared test, Fishers exact test and Students t-test will perform for statistical analysis of differences in gender, site, jaw and tooth type. The statistical significance for all the analyses was set at $(\mathrm{P}<0.05)$

\section{RESULTS}

Evaluation of the panoramic radiographs of 5100 dental patients showed a total of 165 patients with at least one missing permanent tooth. Results are represented in Figure 2. The majority of the patients(99) had two congenitally missing permanent teeth $(60 \%)$, followed by one congenitally missing permanent tooth (57patient) (34.55\%). More than three missing teeth were observed among nine patients $(5.5 \%)$. When the percentage of patients with tooth a genesis was compared to the number of missing teeth ,a statistically significant difference was noted, indicating that tooth agenesis with one or two missing teethe is more common than multiple missing teeth $(\mathrm{p}<0.05)$.

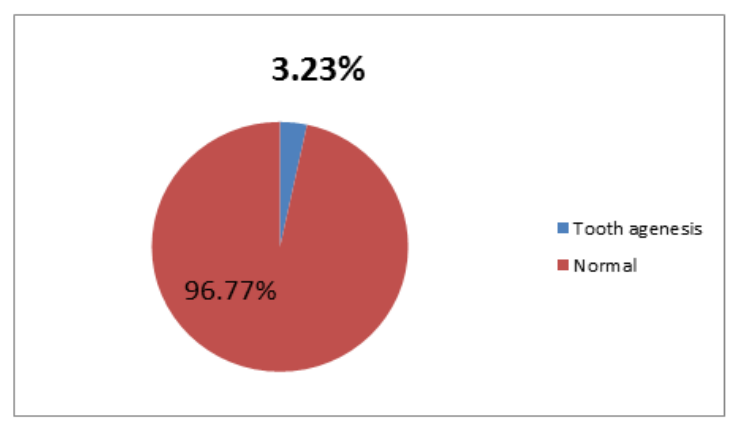

Fig-2: Percentage of tooth agenesis (CMT) 
Out Of 165 patients with tooth agenesis, female patients were $136(82.3 \%)$ and male patients were 29 $(17,7 \%)$. The prevalence of congenitally missing teeth in males was $2.23 \%$ and in females was $3.58 \%$ Table (2). Chi-square test revealed significant association between gender and tooth agenesis $(\mathrm{P}<.05)$ Table $(3)$.

Table-2: Prevalence of tooth agenesis according to gender

\begin{tabular}{|l|l|l|}
\hline Gender & Tooth agenesis & Normal \\
\hline Male & $2.23 \%$ & $97.77 \%$ \\
\hline Female & $3,58 \%$ & $96.42 \%$ \\
\hline
\end{tabular}

Table-3: Association between agenesis and Gender

\begin{tabular}{|l|l|l|l|l|}
\hline Gender & Normal\% & Agenesis\% & Total\% & p-value \\
\hline Male & $1271(97.77)$ & $29(2.23)$ & $1300(100)$ & \\
\hline Female & $3664(96.42)$ & $136(3.58)$ & $3800(100)$ & \\
\hline Total & $4935(96.77)$ & $165(3.23)$ & $5100(100)$ & $<0.05$ \\
\hline
\end{tabular}

Another table issue is the fact that bilateral toothagenes is more common than for the unilateral and was found in $6545 . \%$ of the affected patients.

Table-4: Prevalence of unilateral and bilateral tooth agenesis

\begin{tabular}{|l|l|l|}
\hline & n(prevalence) & p-value \\
\hline Unilateral & $57(1.12)$ & \\
\hline Bilateral & $108(2.11)$ & \\
\hline Total & $165(3.23)$ & $<0.05$ \\
\hline
\end{tabular}

293 absent teeth were reported. The maxillary lateral incisor was found to be the most affected tooth (78 patients- 113 missing teeth), followed by the mandibular second premolar (60 patients- 112 missing teeth), maxillary second premolar (26 patients- 46 missing teeth), mandibular lateral incisor (10patients20 missing teeth), and the maxillary canine (1patients2 missing teeth). The percentage of dental agenesis varied according to the tooth type (Figure 3) and (Figure 4). - 34.29\% of the patients had at least one missing mandibular second premolar $-38.22 \%$ of the absent teeth were mandibular second premolars; $44.58 \%$ of the patients had at least one missing maxillary lateral incisor - $38.56 \%$ of the absent teeth were maxillary lateral incisors; - $14.85 \%$ of the patients had at least one missing maxillary second premolar $15.70 \%$ of the absent teeth were maxillary second premolars; - $5.7 \%$ of the patients had at least one missing mandibular lateral incisor $-6.82 \%$ of the absent teeth were mandibular lateral incisors; $-0.57 \%$ of the patients had at least one missing maxillary canine $0.7 \%$ of the absent teeth were maxillary canines.

In terms of locations, congenitally missing maxillary lateral incisors in 64 patients were found in the right side and 49 in the left side, While congenitally missing lateral incisor was found bilaterally among 35 patients. Maxillary second premolar a genesis in 23 patients was found in the right side and 23 in the left side, while maxillary second premolar agenesis was found bilaterally in 20 patients. Maxillary canine agenesis was found only in one patient bilaterally. Mandibular lateral incisor agenesis was found in 10 patients bilaterally. Mandibular second premolar agenesis in 56 patients was found in the right side and 56 in the left side, while mandibular second premolar agenesis was found bilaterally among 52 patients.

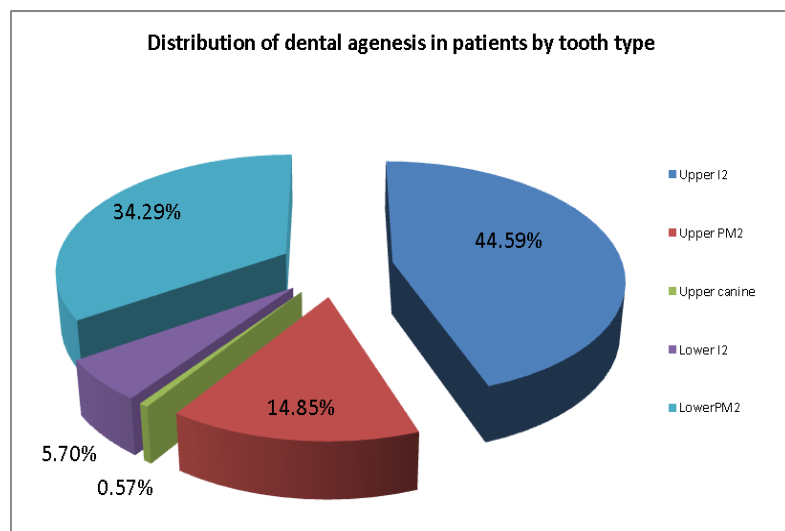

Fig-3: Distribution of dental agenesis in patients according to tooth type

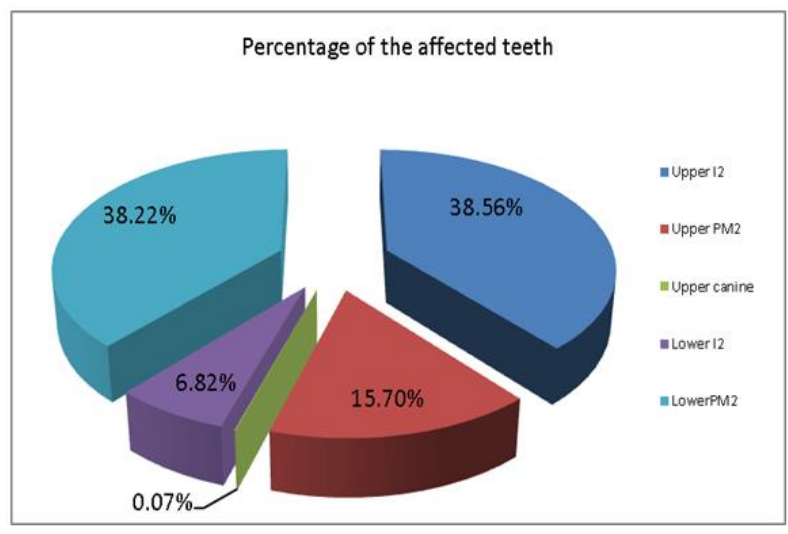

Fig-4: Percentage of affected teeth

Table-5: Distribution of CMT in the maxillary and mandibular arches according to location and gender

\begin{tabular}{|l|l|l|l|l|l|l|}
\hline \multicolumn{3}{c}{ Maxillary arch } & \multicolumn{3}{c|}{ Mandibular ach } \\
\hline Congenitally missing teeth & male & female & total & male & female & total \\
\hline Right lateral incisor & 12 & 52 & 64 & 0 & 10 & 10 \\
\hline Left lateral incisor & 8 & 41 & 49 & 0 & 10 & 10 \\
\hline Right canine & 0 & 1 & 1 & 0 & 0 & 0 \\
\hline Left canine & 0 & 1 & 1 & 0 & 0 & 0 \\
\hline Right $2^{\text {nd }}$ premolar & 2 & 21 & 23 & 14 & 42 & 56 \\
\hline Left $2^{\text {nd }}$ premolar & 2 & 21 & 23 & 14 & 42 & 56 \\
\hline
\end{tabular}


Also, there were $161 \mathrm{CMT}$ in the maxillary arch and $132 \mathrm{CMT}$ in the mandibular arch figure (5).

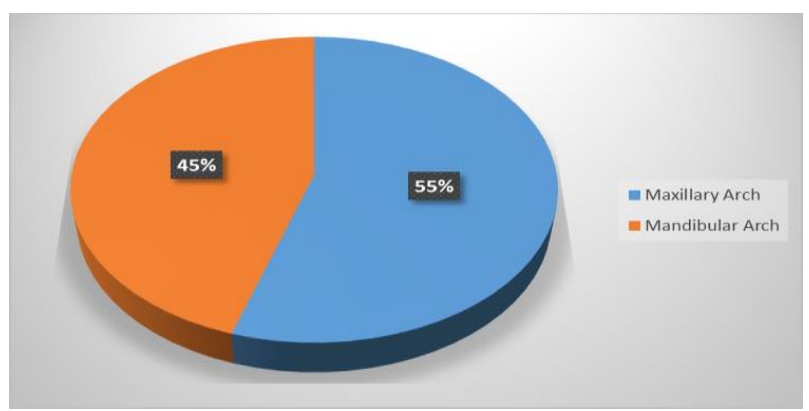

Fig-5: Distribution of CMT in the maxillary and mandibular arch

Of the 293 CMT, 154 were in the right side and139 were in the left side figure (6).

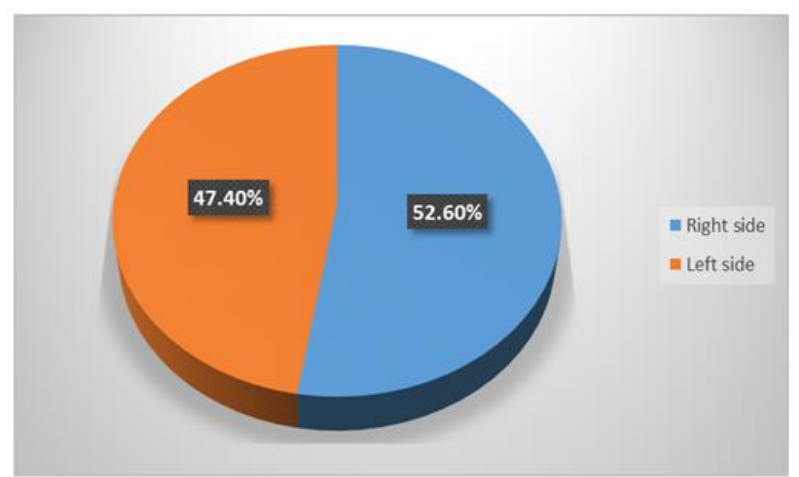

Fig-6: Distribution of CMT in the right and left side

The prevalence of anomalies that were coexisted along with teeth agenesis was $(40 \%)$ in 60 patients. From all co-existing anomalies, impaction was the most common among $29(17.58 \%)$ patients followed by microdontia among $27(16.37 \%)$ patients. Other anomalies present were transposed teeth among 4 $(2.43 \%)$ patients, ectopic eruption among $4(2.43 \%)$ patients and supernumerary among $2(1.21 \%)$ patients. The other anomalies are summarized in Table (6).

Table-6: Prevalence and types of other anomalies

\begin{tabular}{|l|l|l|}
\hline $\begin{array}{l}\text { Characteristic } \\
\text { Gender }\end{array}$ & (Frequency (n & $\%$ Percent \\
\hline No anomalies & 99 & $\% 60$ \\
\hline Impaction & 29 & $\% 17.58$ \\
\hline Microdontia & 27 & $\% 16.37$ \\
\hline eruption Ectopic & 4 & $\% 2.43$ \\
\hline Transposition & 4 & $\% 2.43$ \\
\hline Supernumerary & 2 & $\% 1.21$ \\
\hline
\end{tabular}

All of the coexisted other anomalies were encountered in the anterior region in association with the maxillary and mandibular lateral incisor agenesis as follow: contralateral one, of which 21 (48.8 per cent) were on the right and 6 (14 per cent) on the left side $(\mathrm{P}>$ $0.05)$.

- 21 of the patients with bilateral maxillary lateral agenesis have also bilateral canine impaction.

- 5 of the patients with unilateral maxillary lateral incisor agenesis have also unilateral canine impaction in the same side

- 3 of the patients with bilateral mandibular lateral incisor agenesis have also bilateral mandibular canine impaction

- 4 of the patients with bilateral maxillary lateral incisor agenesis have also bilateral buccally erupted canine

- 4 of the patients with maxillary lateral incisor agenesis have also canine transposition with first premolar

- 2 of the patients with bilateral maxillary lateral incisor agenesis have also supernumerary tooth between central incisors (mesiodens)

- Anothe notable issue was that the primary tooth retention was found among $61(35 \%)$ of the patients with congenitally missing teeth. The percentage of primary tooth retention according to tooth type was as follow:

- In those patients with developmental absence of the upper lateral incisors $(n=78)$, the primary tooth was retained in 14 cases (20 per cent). This retention was bilateral in seven individuals $(8.9 \%)$, and unilateral in the other seven, with the upper right being retained in four $(5.1 \%)$, and the upper left in three $(3.8 \%)$.

- In those patients with mandibular lateral incisor agenesis $(n=10)$, the primary tooth was retained in 3 patients. This retention was bilaterally among the three patients.

- In those patients with mandibular second premolar agenesis $(n=60)$, the primary tooth was retained in $40(66.7 \%)$. This retention was bilateral in 38 patients $(63.3 \%)$ and unilateral in the other two (3.4\%), with the lower right being retained in one patient and the lower left in one patient.

- In those patients with maxillary second premolar agenesis $(n=26)$, the primary tooth was retained in 4 patients $(15.38 \%)$. This retention was bilateral among the 4 patients.

- 27 of the patients with unilateral maxillary lateral incisor agenesis (43) have also a microdontia of the 
Ziad A. N. Musaed et al; Saudi J Oral Dent Res, Oct 2019; 4(10): 704-715

\section{Representive panoramic radiographs}

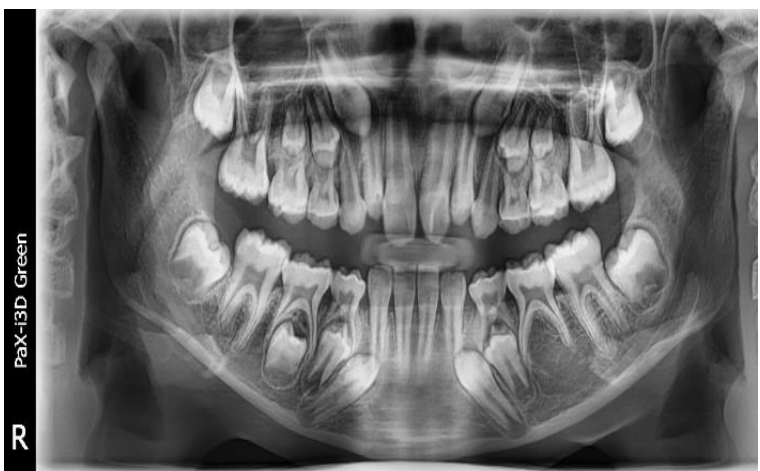

A) Panoramic radiograph depicating unilatetal mandibular 2nd premolar agenesis

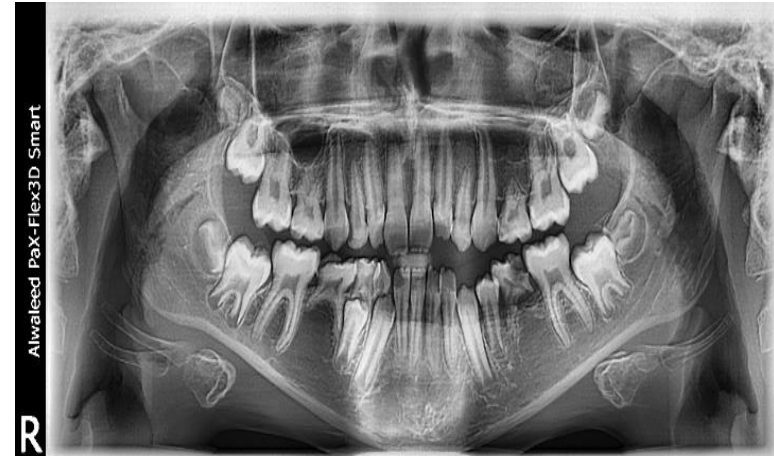

B) Panoramic radiograph depicating bilateral maxillary and mandibular second premolar agenesis

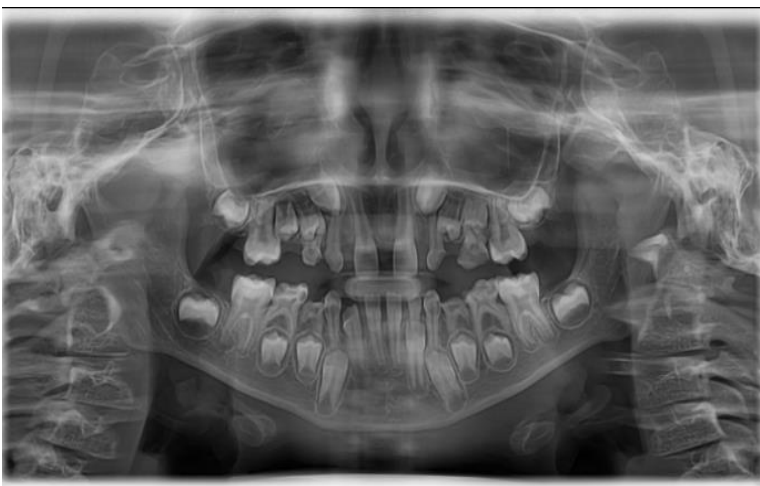

C) Panoramic radiograph depicating maxillary lateral incisors agenesis associated with diastema and canine impaction

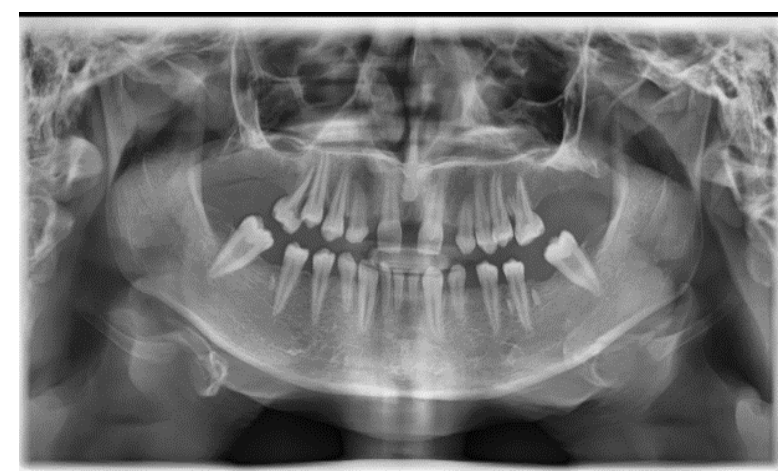

D) Panoramic radiograph depicating multiple tooth agenesis in the maxillary and mandibular arches

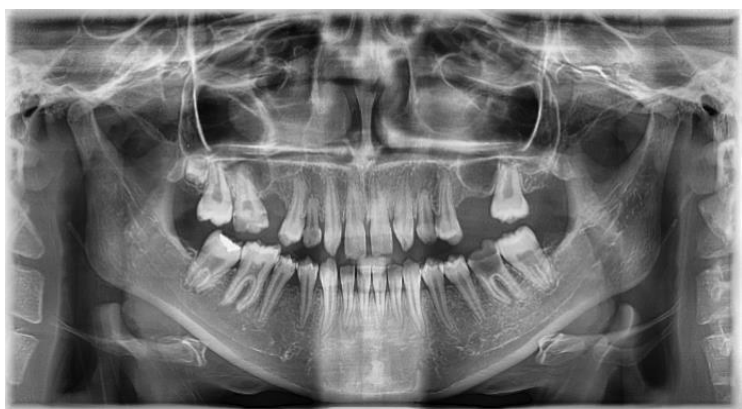

E) Panoramic radiograph depicating bilateral maxillary lateral incisor and 2nd premolar agenesis

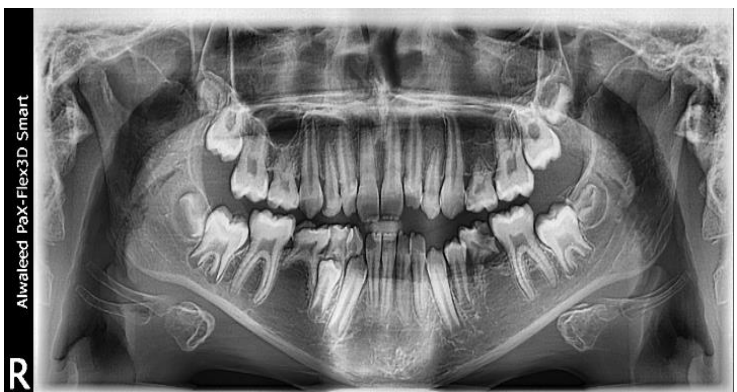

F) Panoramic radiograph depicating bilateral maxillary and mandibular 2nd premolar agenesis

Fig-7: Panoramic radiographs depicating tooh agenesis

\section{DISCUSSION}

Oral health plays a crucial role in public health [35]. It has strong biological, psychological and social projections, because it affects aesthetics and communications, and the quality of life is affiliated with oral health status [36]. Dental treatments are rather expensive health services and the combination of different modalities such as orthodontic, prosthodontics, and surgical treatments can put a heavy burden on the average family's health budget. Some frequent dental anomalies need quite expensive treatments. One of them is congenitally missing teeth (CMT), congenital absence of teeth, congenital dental aplasia, or dental agenesis [1].

In orthodontics, hypodontia due to agenesis of certain teeth greatly affects a patients function and aesthetic, especially the upper lateral incisors [30].The incidence of hypodontia in the anterior segments requires great need for orthodontic and prosthodontics treatment. Orthodontic treatment should be in harmony with modern medical thought: "It is better to prevent than to cure". We should not treat the symptom; we should treat the cause. Therefore, early recognition of a tooth agenesis is helpful in order to provide good treatment and prevent a developing malocclusion, also with early detection of hypodontia, alternative treatment modalities can be planned and performed with multidisciplinary team approach restoring the esthetic and function [30]. 
In human dentition, maxillary and mandibular incisors are important from an esthetic as well as a functional point of view when they are present in their normal position. One only needs to look at the contribution of the maxillary lateral incisor to the upper face to sense its importance; its absence cause either diastema between centrals, midline shifting, collapsing anterior maxilla leading to class III skeletal relationship, or causing canine impaction which complicate the situation.

Although the percentage of dental agenesis has been reported in many countries, there has been no data published among Yemeni population about the prevalence of tooth agenesis in the permanent dentition. The present study aimed to determine the overall prevalence of CMT in a sample of Yemeni dental patients at Sana'a, Ibb, and Thamar provinces.

The prevalence of tooth agenesis ranged between $2.66 \%$ and $12.6 \%$ (Table 7 ). However, in the present study, the overall prevalence of tooth agenesis was found to be $3.23 \%$. The observed discordance can be attributed to the genetic and racial differences as well as to the sample size of the examined group. Many authors have suggested that it is more appropriate to compare any such data obtained from a specific group to those drawn from other similar ethenic groups of the same area living in different geographic locations. Since our current data were drawn from Yemenis - inhabitants of the Arabian Peninsula - who are considered, historically speaking, to be the origin of all Arabs, we believed that it would be interisting to compare our results with those of other arabian groups living in the same geographic area and then with other racial groups.

It was interesting to find out a real gradual increase in dental agenesis prevalence from south to north, with Yemenis (south part) showing the lowest tooth agenesis prevalence, followed by Saudis (middle part) and Jordanians (north part). In the literature, the lowest percentages of tooth agenesis were reported by Abu-Affan and serorin asample of 2401 sudanese (2.6\%), Al-Emran [37] in a sample of 500 Saudis male children (4\%) and by Celikoglu et al. [9] who examined Turkish orthodontic patients $(4.6 \%)$, and the highest percentages of tooth agenesis were reported by Behr et al. [38] in a sample of 1353 Germany orthodontic patients, and by Young HA [39] in a sample of 3055 Korean orthodontic patients $(11.3 \%)$.

The present study analyzed the prevalence of tooth agenesis in a large sample of dental and orthodontic patients. The nature of the examined subjects usually influences prevalence rates of the examined anomalies. The prevalence reported by this study falls short of most of the published data from studies on tooth agenesis in orthodontic populations. Higher prevalence rates have been reported in the latter because patients with hypodontia are usually more motivated to seek orthodontic treatment to restore their dental and/or facial aesthetics $[13,35,38,40,18]$.

A high prevalence of CMT was found in the maxillary arch compared to the mandibular arch; this was coincident to the findings of Vahid-Dastjerdi et al. [7] who obtained a higher prevalence of CMT in the maxillary arch among Iranian orthodontic patients. Also this result corresponds with the analysis performed by Peker et al. [42], as well as Fekonja [5] and Wong et al. [41] who found missing teeth considerably more frequently in the upper arch than in the lower arch in orthodontic patients. However, Kirzioglu [43] found more missing teeth in the mandible than in the maxilla. Gomes [44] found maxillary hypodontia in 59.2\% of patients and in the mandible of $40.8 \%$ with an overall ratio of $1.45: 1$ in orthodontic patients.

The prevalence of CMT in the right side of the jaw was found to be more frequent than in the left side in our study; this was in agreement with the findings of Ferkonja [5] who reported a higher prevalence on the right side of the jaw among 212 orthodontically treated children. No gender dimorphism in the prevalence of CMT was reported among different populations. In the present study, this prevalence was statistically different. These findings dis coincide with those of previous studies. However others recorded a high prevalence of hypodontia among females. On the other hand Nganga et al. reported that in Kenyan population hypodontia was more predominant among males than females.

Bilateral agenesis manifested a frequency of $2.11 \%$ in our study. The most common bilaterally missing teeth were the mandibular lateral incisor and the maxillary second premolar. Goya et al. [17] found that symmetry of congenitally missing teeth was predominant $(74.6 \%)$, and Kirzioglu et al. [43] observed that bilaterally missing teeth was $73.2 \%$. Moreover, symmetrical hypodontia was predominant, being found in both the contralateral and antagonistic quadrant, possibly suggesting a strong genetic pattern of hypodontia. It was demonstrated also that permanent tooth agenesis, maxillary lateral incisor microdontia, palatally displaced canines, and distoangulation of mandibular second premolars were frequently associated with maxillary lateral incisor agenesis, providing additional evidence of a genetic interrelationship in the causes of hypodontia [45]. Moreover, a significant decrease in maxillary transversal and sagittal size was demonstrated in patients with dental agenesis [46].

It was claimed that missing maxillary incisors were significantly more frequent in girls; however, the relationship could not be explained. However, it is emphasized that the cause may be dimorphism based on the sex origin occurring during growth and development [47]. In our study, the lack of upper incisors was found to be significantly higher in girls, 
supporting these results. Interestingly, the lack of bilateral lateral incisors in girls was also found to be quite high. It is also claimed that the lack of bilateral upper incisors is due to genetic origin while unilateral missing is due to a developmental anomaly, therefore, the tooth formed on one side is usually conical or microdontia [48]. Our study also supports these results.

The most frequently missing tooth in our study was the maxillary lateral incisor. This finding was in agreement with the findings of studies in the Malaysian [8], Turkish [9], Romanian [10], Spanish [11], and American [12] populations. In contrast to our finding, the mandibular second premolars were the most common in the the Jordanian [13], Iraqi [14], Indian [15] and European [6] populations. However, a higher incidence of missing mandibular incisors is observed in Chinese [16], Korean [17], and Japanese [18] populations than in Caucasian populations.

In this study, canine impaction was seen in 29 patients while microdontia was found in 27 patients. It has been reported that the existence of associations between various dental anomalies is clinically relevant, as early diagnosis of one dental anomaly might indicate an increased risk for others [49].A general consensus has been reached in finding that there exists a significant correlation between tooth agenesis(maxillary lateral) and either maxillary canine impaction or microdontia of maxillary lateral. Sacerdoti and Baccetti [50] reported an increased prevalence of maxillary canine impaction in a sample of subjects with maxillary lateral incisor agenesis, compared with a control group. Moreover, Camilleri [52] observed 106 subjects with maxillary canine impaction and stated a strong connection with hypodontia. The most important findings of this study were about the association between tooth agenesis and maxillary canine impaction: it was statistically attributable only at the lack of maxillary lateral incisor, whereas the mandibular second premolar or other types of agenesis did not show any significant association. The presence of a substantial relationship between agenesis of maxillary lateral incisors and maxillary canine impaction could be explained by the guidance theory. If the lateral incisor is absent, the canine will not find the guidance that would enable it to descend along its normal eruption path and move down in a more palatal path until it comes close to the periosteum of the medial aspect of the alveolar process [52]. According to our results, several studies reported that maxillary canine impaction and agenesis of lateral incisors could be a strong predictor of maxillary canine impaction [53].
The etiology and exact mechanism of dental agenesis is still unclear, although, number of genetic and environmental factors has been suggested. One propsed mechanism for tooth agenesis occurence is a combination of genetic susceptibility and environment factors. Thus, in the presence of gene defect (defects in the genes responsible for tooth formation), the environmental factors can affect tooth formation process, resulting in tooth agenesis and other dental anomalies.

Ovarian cancer, known as the silent killer in women, is difficult to diagnosis due to a lack of effective early screening markers for this disease. Without improvements in the current early detection protocols, over $75 \%$ of women diagnosed with ovarian cancer will be identified in late stage of disease with a significantly reduced chance of survival. Lopes et al.[54] reported that anomalies of the teeth may be present in many diseases and dentists may be the first to notice them particularly through the preventive children screening programmes. Previous studies have demonstrated that the genes that control the tooth development may have an important function in other organs and cancer diseases [55, 56]. Zhai et al. [57] indicated in their study that $\beta$-catenin and TCF plays a vital role in the activation of AXIN2 expression in colon and ovarian cancer cells. Lammi et al. [55] reported about evidence of the expression of association AXIN2 in colorectal tissue leading to carcinoma and hypodontia in a Finnish family. It is interesting that one gene mutation can cause tooth agenesis and predispose to colorectal cancer. Therefore, by estimation of the prevalence of hypodontia, we can predict the degree of danger and susceptibility to developing cancer in those patients having hypodontia (it serve as a marker for potential risk of cancer), therefore the dentists should take this information in to their considerations, especially when dealing with women. Therefore, the community health programs should be targeted to get rid of the possible causes and risk factors of hypodontia. Furthermore, Womens with hypodontia may have or may develop ovarian cancer speccially if they have chronic inflammations in the ovarian endothelium, therfore, the dentist can help in the diagnosis and/or pevention of cancer development.

The knowledge gained from this study will assist dental practitioners to better understand tooth agenesis and design treatment plans that address the esthetic and functional needs of affected individuals and to improve quality of the provided treatment outcome 
Ziad A. N. Musaed et al; Saudi J Oral Dent Res, Oct 2019; 4(10): 704-715

Table-6: Prevalence of CMT of permanent dentition in different studies

\begin{tabular}{|l|l|l|l|l|}
\hline Author & country & sample size & prevalence & The most common CMT \\
\hline Behr[38] & Germany & 1353 & $\% 12.6$ & \\
\hline Muller [12] & America & 14,940 & $\% 3.5$ & maxillary lateral incisor \\
\hline Gonzallez[58] & Portugal & 2888 & $6.1 \%$ & mandibular 2nd premolar \\
\hline Mammon [59] & Jordan & 3660 & $8.85 \%$ & \\
\hline Young Ho [39] & korea & 3055 & $11.3 \%$ & mandibular 2nd premolar \\
\hline Vahid [7] & Iran & 1751 & $9.1 \%$ & maxillary lateral incisor \\
\hline Cantekin[48] & Turkey & 1291 & $\% 6.2$ & \\
\hline Tallon [11] & Spain & 1518 & $9.48 \%$ & \\
\hline Celikoglu[9] & Turkey & 3341 & $4.6 \%$ & maxillary lateral incisor \\
\hline Mani [8] & Malysian & 834 & $\% 4.2$ & maxillary lateral incisor \\
\hline Gomes[44] & Brazil & 1049 & $6.3 \%$ & maxillary lateral incisor \\
\hline Al-Ajwadi [14] & Iraq & 389 & & maxillary lateral incisor \\
\hline Sisman [60] & Turkey & 2413 & $7.54 \%$ & maxillary lateral incisor \\
\hline Albashaireh and Khater, [61] & Jordan & 1045 & $5.5 \%$ & mandibular 2nd premolar \\
\hline Goren [62] & Israel & 226 & $5.3 \%$ & maxillary lateral incisor \\
\hline Fekonja[5] & Slovenia & 212 & $11.3 \%$ & maxillary lateral incisor \\
\hline Goya[17] & Japan & 2072 & $9.4 \%$ & mandibular2nd premolar \\
\hline Ng'ang'a et al. [63] & kenya & 615 & $6.3 \%$ & mandibular 2nd premolar \\
\hline Bernadette[10] & Romania & 946 & $\% 06.34$ & maxillary lateral incisor \\
\hline Al-Emran[37] & KSA & 500 & $4 \%$ & mandibular2nd premolar \\
\hline Nordgarden[57] & Norway & 9532 & $\% 4.5$ & \\
\hline Davis [16] & China & 1093 & $6.9 \%$ & mandibular incisor \\
\hline Rolling[64] & Denmark & 8138 & $7.39 \%$ & mandibular 2nd premolar \\
\hline Magnussom[65] & Iceland & 1116 & $6.7 \%$ & mandibular 2nd premolar \\
\hline Ajami [15] & India & 600 & $\% 10.6$ & maxillary lateral incisor \\
\hline Al-Moherat [13] & Jordan & 1726 & $\% \mathbf{0} \%$ & maxillary lateral incisor \\
\hline Affan[14] & Sudan & 2401 & $2.66 \%$ & mandibular lateral incisor \\
\hline Ziad Ali & Yemen & 5100 & $3.23 \%$ & maxillary laterl incisor \\
\hline & & & \\
\hline
\end{tabular}

\section{CONCLUSION}

The prevalence of congenitally missing teeth was $3.21 \%$ and there was significant difference in the prevalence among male and females. Agenesis occurred more bilaterally than unilaterally. The most common missing tooth was the maxillary lateral incisor. Maxillary canine impaction and maxillary lateral incisor microdontia have a high significant association with maxillary lateral incisor agenesis.

Dentists or pediatric dentists are the first to diagnose congenital tooth agenesis. Determining tooth agenesis in dentition early increases the potential for functional, aesthetic and stable outcomes. However, considering that hypodontia is often associated with a familial, syndromic or non-syndromic condition, the medical conditions related with the situation can also be diagnosed during the routine examinations of pediatricians. At this point, in cases where congenital tooth agenesis is considered, pediatricians should work in cooperation with dentists or pediatric dentists. Moreover, in addition to hundreds of syndromic conditions related with hypodontia, non-syndromic cases should also be investigated in terms of familial history and dental anamnesis should be obtained, and if needed, contact with a pediatric dentist might be helpful in early diagnosis. Future studies including larger samples are needed to evaluate the etiology of hypodontia and tooth agenesis in Yemen.

\section{REFERENCES}

1. De Coster, P. J., Marks, L. A., Martens, L. C., \& Huysseune, A. (2009). Dental agenesis: genetic and clinical perspectives. Journal of Oral Pathology \& Medicine, 38(1), 1-17.

2. Valle, A. L. D., Lorenzoni, F. C., Martins, L. M., Valle, C. V. M. D., Henriques, J. F. C., Almeida, A. L. P. F. D., \& Pegoraro, L. F. (2011). A multidisciplinary approach for the management of hypodontia: case report. Journal of Applied Oral Science, 19(5), 544-548.

3. Rakhshan, V., (2015). Congenitally missing teeth (hypodontia): A review of the literature concerning the etiology, prevalence, risk factors, patterns and treatment. Dent. Res. J. 12, 1-13.

4. Temilola, D.O., Folayan, M.O., Fatusi, O., Chukwumah, N.M., Onyejaka, N., .Oziegbe, E.(2014). The prevalence, patern and clinical presentaton of developmental dental hard-tssue anomalies in children with primary and mix dentton from Ile-Ife, Nigeria. BMC Oral Health, 14: 125.

5. Fekonja, A. (2005). Hypodontia in orthodontically treated children. The European Journal of Orthodontics, 27(5), 457-460. 
6. Polder, B. J., Van't Hof, M. A., Van der Linden, F. P., \& Kuijpers-Jagtman, A. M. (2004). A metaanalysis of the prevalence of dental agenesis of permanent teeth. Community dentistry and oral epidemiology, 32(3), 217-226.

7. Vahid-Dastjerdi, E., Borzabadi-Farahani, A., Mahdian, M., \& Amini, N. (2010). Non-syndromic hypodontia in an Iranian orthodontic population. Journal of oral science, 52(3), 455461.

8. Mani, S. A., Mohsin, W. S. Y., \& John, J. (2014). Prevalence and patterns of tooth agenesis among Malay children. Southeast Asian Journal of Tropical Medicine and Public Health, 45(2), 490.

9. Celikoglu, M., \& Kamak, H. (2011). Patterns of third-molar agenesis in an orthodontic patient population with different skeletal malocclusions. The Angle Orthodontist, 82(1), 165169.

10. Kerekes-Máthé, B., Mártha, K., \& Székely, M. (2013). Prevalence and Characteristics of Tooth Agenesis in Permanent Dentition of Subjects from Tîrgu Mureş. Acta Medica Marisiensis, 59(4).

11. Walton, V. T., Nieminen, P., Arte, S., Lobato, P. C., Torrent, J. M. U., \& Céspedes, M. C. M. (2010). An epidemiological study of dental agenesis in a primary health area in Spain: estimated prevalence and associated factors. Medicina oral, patología oral y cirugía bucal. Ed. inglesa, 15(4), 5.

12. Muller, T. P., Hill, I. N., Petersen, A. C., \& Blayney, J. R. (1970). A survey of congenitally missing permanent teeth. The Journal of the American Dental Association, 81(1), 101-107.

13. Al-Moherat, F. H., Al-Ebrahim, H. M., Alshurman, I. S., Shudefat, N., \& Al-Tawareh, Y. (2009). Hypodontia in orthodontic patients in Southern Jordan. Pak Oral Dent J, 29, 45-48.

14. Al-Ajwadi, S. A. M. (2009). An orthopantomographic study of hypodontia in permanent teeth of Iraqi patients. MUSTANSIRIA DENTAL JOURNAL, 6(2), 139-144.

15. Ajami, B., Shabzendedar, M., Afzal Agaee, M., \& Mehrjerdian, M. (2019). Prevalence and pattern of congenital missing teeth in a group of Iranian adolescents. Journal of Dentistry, 10(Supplement 2010), 40-44.

16. Davis, P. J. (1987). Hypodontia and hyperdontia of permanent teeth in Hong Kong schoolchildren. Community Dentistry and Oral Epidemiology, 15(4), 218-220.

17. Chung, C. J., Han, J. H., \& Kim, K. H. (2008). The pattern and prevalence of hypodontia in Koreans. Oral diseases, 14(7), 620-625.

18. Endo, T., Ozoe, R., Kubota, M., Akiyama, M., \& Shimooka, S. (2006). A survey of hypodontia in Japanese orthodontic patients. American Journal of Orthodontics and Dentofacial Orthopedics, 129(1), 29-35.
19. Brook, A. H., Jernvall, J., Smith, R. N., Hughes, T. E., \& Townsend, G. C. (2014). The dentition: the outcomes of morphogenesis leading to variations of tooth number, size and shape. Australian dental journal, 59, 131-142.

20. Kaste, S. C., Hopkins, K. P., Jones, D., Crom, D., Greenwald, C. A., \& Santana, V. M. (1997). Dental abnormalities in children treated for acute lymphoblastic leukemia. Leukemia, 11(6), 792.

21. Svinhufvud, E., Myllärniemi, S., \& Norio, R. (1988). Dominant inheritance of tooth malpositions and their association to hypodontia. Clinical Genetics, 34(6), 373-381.

22. Sönmez, I., \& Oba, A. A. (2007). Isolated oligodontia: Case report. Cumhuriyet Dent J, 10(2), 108-12.

23. Larmour, C. J., Mossey, P. A., Thind, B. S., Forgie, A. H., \& Stirrups, D. A. (2005). Hypodonhia--A retrospective review of prevalence and etiology. Part I. Quintessence international, 36(4).

24. Pirinen, S., Kentala, A., Nieminen, P., Varilo, T., Thesleff, I., \& Arte, S. (2001). Recessively inherited lower incisor hypodontia. Journal of medical genetics, 38(8), 551-556.

25. Ahmad, W., Brancolini, V., Ul Faiyaz, M. F., Lam, H., ul Haque, S., Haider, M., ... \& Zegarelli, D. J. (1998). A locus for autosomal recessive hypodontia with associated dental anomalies maps to chromosome 16q12. 1. American journal of human genetics, 62(4), 987.

26. Brook, A. H. (2009). Multilevel complex interactions between genetic, epigenetic and environmental factors in the aetiology of anomalies of dental development. Archives of oral biology, 54, S3-S17.

27. Ben-Bassat, Y., \& Brin, I. (2009). Skeletal and dental patterns in patients with severe congenital absence of teeth. American Journal of Orthodontics and Dentofacial Orthopedics, 135(3), 349-356.

28. Scarel, R. M., Trevilatto, P. C., Di Hipólito Jr, O., Camargo, L. E., \& Line, S. R. (2000). Absence of mutations in the homeodomain of the MSX1 gene in patients with hypodontia. American journal of medical genetics, 92(5), 346-349.

29. Stockton, D. W., Das, P., Goldenberg, M., D'Souza, R. N., \& Patel, P. I. (2000). Mutation of PAX9 is associated with oligodontia. Nature genetics, 24(1), 18.

30. Goodman, J. R., Jones, S. P., Hobkirk, J. A., \& King, P. A. (1994). Hypodonta 1: Clinical features and the management of mild to moderate hypodontia. Dental Update, 21, 381-384.

31. Stamatiou, J., \& Symons, A. L. (1991). Agenesis of the permanent lateral incisor: distribution, number and sites. J Clin Pediatr Dent, 15(4), 244-6.

32. Hobson, R. S., Carter, N. E., Gillgrass, T. J., Jepson, N. J. A., Meechan, J. G., Nohl, F., \& Nunn, J. H. (2003). The interdisciplinary management of hypodontia: the relationship between an 
interdisciplinary team and the general dental practitioner. British dental journal, 194(9), 479.

33. Aktan, A. M., Kara, I. M., Şener, İ., Bereket, C., Ay, S., \& Çiftçi, M. E. (2010). Radiographic study of tooth agenesis in the Turkish population. Oral Radiology, 26(2), 95-100.

34. Park, J. H., Tai, K., \& Hayashi, D. (2010). Tooth autotransplantation as a treatment option: a review. Journal of Clinical Pediatric Dentistry, 35(2), 129-135.

35. Altug-Atac, A. T., \& Erdem, D. (2007). Prevalence and distribution of dental anomalies in orthodontic patients. American Journal of Orthodontics and Dentofacial Orthopedics, 131(4), 510-514.

36. Gift, H. C., \& Atchison, K. A. (1995). Oral health, health, and health-related quality of life. Medical care.

37. Al-Emran, S. (1990). Prevalence of hypodontia and developmental malformation of permanent teeth in Saudi Arabian schoolchildren. British Journal of Orthodontics, 17(2), 115-118.

38. Behr, M., Proff, P., Leitzmann, M., Pretzel, M., Handel, G., Schmalz, G., ... \& Koller, M. (2010). Survey of congenitally missing teeth in orthodontic patients in Eastern Bavaria. The European Journal of Orthodontics, 33(1), 32-36.

39. Kim, Y. H. (2010). Investigation of hypodontia as clinically related dental anomaly: prevalence and characteristics. ISRN dentistry, 2011.

40. Durrani, O. K., Zulfiqar, K., Bashir, U., \& Shamsher, M. (2010). Prevalence of tooth agenesis in orthodontic patients at Islamic International Dental Hospital. Pakistan Orthodontic Journal, 2(2), 48-51.

41. Wong, A. T. Y., McMillan, A. S., \& McGrath, C. (2006). Oral health-related quality of life and severe hypodontia. Journal of Oral Rehabilitation, 33(12), 869-873.

42. Peker, I., Kaya, E., \& Darendeliler-Yaman, S. (2009). Clinic and radiographical evaluation of non-syndromic hypodontia and hyperdontia in permanent dentition. Med Oral Patol Oral Cir Bucal, 14(8), e393-7.

43. Kırzıoğlu, Z., Köseler Şentut, T., Özay Ertürk, M. S., \& Karayılmaz, H. (2005). Clinical features of hypodontia and associated dental anomalies: a retrospective study. Oral diseases, 11(6), 399-404.

44. Gomes, R. R., Da Fonseca, J. A. C., Paula, L. M., Faber, J., \& Acevedo, A. C. (2009). Prevalence of hypodontia in orthodontic patients in Brasilia, Brazil. The European Journal of Orthodontics, 32(3), 302-306.

45. Garib, D. G., Alencar, B. M., Lauris, J. R. P., \& Baccetti, T. (2010). Agenesis of maxillary lateral incisors and associated dental anomalies. American Journal of Orthodontics and Dentofacial Orthopedics, 137(6), 732-e1.

46. Laganà, G., Lombardi, C. C., Franchi, L., \& Cozza, P. (2011). Tooth agenesis: dento-skeletal characteristics in subjects with orthodontic treatment need. European journal of paediatric dentistry: official journal of European Academy of Paediatric Dentistry, 12(1), 17-20.

47. Pinho, T., Tavares, P., Maciel, P., \& Pollmann, C. (2005). Developmental absence of maxillary lateral incisors in the Portuguese population. The European Journal of Orthodontics, 27(5), 443-449.

48. Stein, P. K., \& Pu, Y. (2012). Heart rate variability, sleep and sleep disorders. Sleep medicine reviews, 16(1), 47-66.

49. Baccetti, T. (1998). A controlled study of associated dental anomalies. The Angle Orthodontist, 68(3), 267-274.

50. Sacerdoti, R., \& Baccetti, T. (2004). Dentoskeletal features associated with unilateral or bilateral palatal displacement of maxillary canines. The Angle Orthodontist, 74(6), 725-732.

51. Camilleri, S. (2005). Maxillary canine anomalies and tooth agenesis. The European Journal of Orthodontics, 27(5), 450-456.

52. Becker, A., \& Chaushu, S. (2015). Etiology of maxillary canine impaction: a review. American journal of orthodontics and dentofacial orthopedics, 148(4), 557-567.

53. Al-Nimri, K. S., \& Bsoul, E. (2011). Maxillary palatal canine impaction displacement in subjects with congenitally missing maxillary lateral incisors. American journal of orthodontics and dentofacial orthopedics, 140(1), 81-86.

54. Lopes, N. N., Petrilli, A. S., Caran, E. M., França, C. M., Chilvarquer, I., \& Lederman, H. (2006). Dental abnormalities in children submitted to antineoplastic therapy. Journal of dentistry for children, 73(3), 140-145.

55. Lammi, L., Arte, S., Somer, M., Järvinen, H., Lahermo, P., Thesleff, I., ... \& Nieminen, P. (2004). Mutations in AXIN2 cause familial tooth agenesis and predispose to colorectal cancer. The American Journal of Human Genetics, 74(5), 1043 1050.

56. Küchler, E. C., Lips, A., Tannure, P. N., Ho, B., Costa, M. C., Granjeiro, J. M., \& Vieira, A. R. (2013). Tooth agenesis association with selfreported family history of cancer. Journal of dental research, 92(2), 149-155.

57. Fekonja, A., Cretnik, A., Zerdoner, D., \& Takac, I. (2015). Hypodontia phenotype in patients with epithelial ovarian cancer. Radiology and oncology, 49(1), 65-70.

58. González-Allo, A., Campoy, M. D., Moreira, J., Ustrell, J., \& Pinho, T. (2012). Tooth agenesis in a Portuguese orthodontics, 10(2), 198-210.

59. Fnaish, M. M., Alawneh, A. M., DA'AMEH, M. D. A., \& Al-Share, A. A. (2011). Dental anomalies in children in North Jordan. Pakistan Oral \& Dental Journal, 31(2).

60. Sisman, Y., Uysal, T., \& Gelgor, I. E. (2007). Hypodontia. Does the prevalence and distribution 
pattern differ in orthodontic patients?. European journal of dentistry, 1(3), 167.

61. Albashaireh, Z. S., \& Khader, Y. S. (2006). The prevalence and pattern of hypodontia of the permanent teeth and crown size and shape deformity affecting upper lateral incisors in a sample of Jordanian dental patients. Community dental health, 23(4), 239.

62. Goren, S., Tsoizner, R., Dinbar, A., Levin, L., \& Brezniak, N. (2005). Prevalence of congenitally missing teeth in Israeli recruits. Refu'at ha-peh veha-shinayim (1993), 22(2), 49-53.
63. $\mathrm{Ng}$ ang a, R. N., \& Ng ang a, P. M. (2001). Hypodontia of permanent teeth in a Kenyan population. East African medical journal, 78(4), 200-203.

64. RØLLING, S. (1980). Hypodontia of permanent teeth in Danish schoolchildren. European Journal of Oral Sciences, 88(5), 365-369.

65. Magnusson, T. E. (1977). Prevalence of hypodontia and malformations of permanent teeth in Iceland. Community Dentistry and Oral Epidemiology, 5(4), 173-178. 